\title{
Treatment Options for Interstitial Lung disease associated with Connective Tissue Disease
}

\author{
Moiz Ehtesham ${ }^{*}$, Fatima Mahmood ${ }^{2}$, Muhammad Asim Shabbir', Ruben-Wende Peredo' \\ 'Department of Internal Medicine, Division of Rheumatology, Albany Medical College, Albany, NY, USA \\ ${ }^{2}$ Department of Medicine, Albany Medical College, Albany, NY, USA
}

Article Info

\section{Article Notes}

Received: August 23, 2021

Accepted: November 15, 2021

\section{*Correspondence:}

Dr. Moiz Ehtesham, Department of Internal Medicine, Division of Rheumatology, Albany Medical College, Albany, NY, USA; Email: ehteshm@amc.edu

(C) 2021 Ehtesham M. This article is distributed under the terms of the Creative Commons Attribution 4.0 International License.

\section{Introduction}

Interstitial lung diseases (ILDs) are the most common pulmonary manifestation of connective tissue diseases (CTDs) and are often the most serious complication. Lung involvement in CTDs is associated with decreased survival rates and higher overall mortality ${ }^{1,2}$. ILD may occur in almost all clinically diagnosed CTDs, but it is more prevalent in systemic sclerosis (SSc) and idiopathic inflammatory myopathies (IIM) $)^{3,4}$. The specific type of ILD can be characterized based on diverse patterns of lung damage seen on high resolution computed tomography (HRCT) and by histopathological analysis of lung biopsy specimens $\mathrm{s}^{5,6}$. These diverse forms of ILD were discussed in previous chapter; they mainly comprise of the following types: usual interstitial pneumonia (UIP), nonspecific interstitial pneumonia (NSIP), diffuse alveolar damage pattern (DAD), lymphocytic interstitial pneumonia (LIP) and organizing pneumonia $(\mathrm{OP})^{7,8}$. Management and treatment options for ILD-CTD have been extensively studied ${ }^{9,10}$, but they still pose a significant challenge due to the paucity of randomized controlled trials (RCTs). It should also be noted that there can be other pulmonary manifestations of CTDs (other than just ILD): these include pleural effusions and bronchiectasis seen with systemic lupus erythematosus (SLE) and rheumatoid arthritis (RA). These effusions are usually bilateral, secondary to pleural inflammation and often resolve with treatment of underlying CTD.

This chapter will describe the various treatment options for ILD-CTDs as described by medical literature while also reviewing ongoing clinical trials. We will go through treatment options for ILDs based on the specific type of CTD.

\section{Disease Specific Treatment}

\section{Systemic Sclerosis (SSc)}

Systemic Sclerosis involves a complex pathogenesis of immune system activation, vascular constriction and increased activity of fibroblasts. The resultant fibrosis can affect every organ of the body and lungs are no exception. ILD is the most prevalent form of pulmonary involvement, and NSIP is the most implicated pattern ${ }^{11,12}$.

Precise criteria for initiation of immunosuppressive regimen for systemic sclerosis- associated interstitial lung disease (SSc-ILD) has not been established yet. The selection of treatment requires knowledge of its toxicity while providing it to those whose disease 
is most likely to progress. In progressive SSc-ILD, the most rapid decline in functional vital capacity (FVC) often occurs within first three years of disease onset and thus timing of therapy becomes crucial in such cases ${ }^{13}$.

\section{Approach to Therapy}

HRCT and pulmonary function test (PFT) results play an important role in determining the timing of initial therapy. Serial PFTs that demonstrate $10 \%$ decline in FVC or $15 \%$ decline in diffusing capacity for carbon monoxide (DLCO) are indications of active disease for which treatment should be considered ${ }^{14}$. Similarly, HRCT demonstrating fibrosis of $20 \%$ or more of lung parenchyma has been associated with increased mortality and thus should guide towards early therapy initiation ${ }^{15,16}$. Additionally, SADL Model (smoking history, Age and \% predicted DCLO) has been shown to estimate 3-year mortality risk in SSc-ILD using readily available clinical parameters and thus can be utilized for therapy initiation conversation ${ }^{17}$.

\section{Initial Therapy}

Mycophenolate mofetil (MMF) and Cyclophosphamide (Суc) have both been used and studied in multiple observational studies and RCTs as initial therapies for SSc-ILD. MMF has been shown to decrease the decline of DLCO, stabilize decrease in FVC and improve respiratory symptoms such as dyspnea and cough ${ }^{18,19}$.

Scleroderma Lung Study II (SLS II) trial showed that the difference of improvement in FVC between MMF and oral Cyc was not significant. Cyc was however associated with higher rate of adverse events such as leukopenia and thrombocytopenia ${ }^{20}$, and thus MMF is often deemed a superior initial agent due to safer profile. On the contrary, MMF can cause gastrointestinal side effects such as cramping, diarrhea and nausea, this may be abated by using enteric coated formulations and dividing dosing regimen.

Cyclophosphamide is also a first line agent used for progressive SSc-ILD and has shown to improve fibrosis and $\mathrm{FVC}^{21,22}$. Both oral and IV Cyc can be used but latter is preferred due to a better side effect profile ${ }^{23}$. Cyc usage is mainly limited due to its adverse effects of hemorrhagic cystitis, leukopenia, and thrombocytopenia. If utilized, its duration is limited to 6-12 months with switching to a less toxic agent for maintenance thereafter.

Low dose glucocorticoid (GC) $(<10 \mathrm{mg} /$ day) has been used with Cyc (in addition to other immunosuppressants) in studies demonstrating Cyc's efficacy, however evidence for its usage is scarce and long term therapy is precluded due to adverse effects related with GC. High dose glucocorticoid are avoided with Cyc due to increased risk of propagating scleroderma renal crisis $^{24}$.

\section{Maintenance Therapy}

Both MMF and Azathioprine (AZA) can be administered for maintenance therapy after an initial round of MMF/ $\mathrm{Cyc}^{25,26}$. MMF is more widely used though due to its proven benefit and efficacy ${ }^{20}$. These agents are utilized until patient experiences a period of clinical stability in respiratory symptoms or adverse effects warrant use of a different therapeutic option. However, the optimal duration of maintenance therapy is yet to be known.

\section{Newer Options}

Elevated levels of Interleukin-6 (IL-6) are associated with progression of SSc-ILD ${ }^{27}$, and earlier in 2021 FDA approved use of tocilizumab in such patients to halt the rate of decline of lung function ${ }^{28}$. Tocilizumab has been shown to decrease rate of FVC change as compared to placebo in clinical trials ${ }^{29,30}$, but there have been no comparisons with MMF directly and thus its usage depends on limitation of MMF or Cyc therapies due to side effects.

Nintedanib is an FDA approved tyrosine kinase inhibitor for idiopathic pulmonary fibrosis(IPF) ${ }^{31}$, it has also been studied in patients with SSc-ILD where it showed considerable improvement in rate of decline of $\mathrm{FVC}^{32}$, however more RCTs need to be undertaken to better assess its efficacy. Nintedanib was approved by the FDA and EMA recently for patients with SSc-ILD and chronic fibrosing ILD (based on results of the SCENSIS and INBUILD trial). Pirfenidone is commonly used to slow progression of IPF, and it has shown improvement in FVC in SSc-ILD patients as well ${ }^{33,34}$. However, larger studies with longer follow-up periods are required for conclusive evidence.

Rituximab has also been studied in a few observational studies and small trials. It shows promising results with improvement in FVC and DLCO compared to standard therapy ${ }^{35-37}$, however its use is limited owing to lack of larger RCTs.

\section{Idiopathic Inflammatory Myopathies (IIM)}

IIM comprise of Dermatomyositis (DM), Polymyositis (PM) and Antisynthetase Syndrome (ASSD). These CTDs involve varying degrees of skeletal muscle inflammation with frequent extra-muscular organ involvement. ILD is the most common extra-muscular manifestation of IIM with NSIP being the most prevalent form ${ }^{38}$, while OP is also frequently seen with IIM.

\section{Approach to Therapy}

As discussed earlier with SSC-ILD, therapeutic approach for IIM-ILD depends on degrees of respiratory symptoms (dyspnea),PFT results, degree and type of fibrosis on HRCT and histopathology, and progression of ILD $^{39}$. It is seen that patients who are positive for melanoma differentiationassociated (MDA) - 5 antibodies have worse survival rates 
compared to those who don't ${ }^{40,41}$, thus necessitating earlier treatment. On the contrary, anti-aminoacyl tRNA synthetase antibodies, a group of myositis-specific autoantibody related to ASSD are associated with a better glucocorticoid response $^{42}$.

\section{Initial Therapy}

Systemic Glucocorticoids form the mainstay of initial therapy for IIM-ILD. Even though there has been no controlled trial demonstrating its efficacy, anecdotal usage has shown that almost half of the patients with DM or PM associated ILD clinically improve with GC. There are also certain clinical/laboratory features that propose high response to GC therapy: younger age, elevated creatine phosphokinase (CPK) levels, PM etiology instead of DM, NSIP rather than UIP on histopathology and ground glass opacities or consolidation on HRCT compared to honeycombing ${ }^{4,43,44}$.

A steroid sparing agent is usually used with initial GC therapy regardless of response to GC.

\section{Second Agent Options}

Exact guidelines to start a second steroid sparing agent have not been yet established but certain clinical features are strong indicators to start a second agent therapy: Impending respiratory failure, antisynthetase syndrome with lung involvement or anti-MDA 5 seropositivity as these are associated with rapidly progressive $\operatorname{ILD}^{45}$.

MMF, AZA, Methotrexate (MTX) and Calcineurin inhibitors have all been used as steroid sparing agents, their choice depends on toxicity of the treatment, agent being used to treat myositis and severity of lung involvement. Both AZA and MMF show comparable efficacies with improvement in HRCT picture, FVC and DLCO, however larger studies are still required to further evaluate the roles of these medications ${ }^{46,47}$. Tacrolimus and Cyclosporine administration have shown improvement in survival and lung parameters in a few studies ${ }^{48,49}$, however lack of larger trials and risk of nephrotoxicity limit their usage to refractory cases. MTX is commonly used as a steroid sparing agent in IIM, however it may cause drug-induced pneumonitis ${ }^{50}$, which may be difficult to differentiate from underlying ILD. Cyclophosphamide may be used for more severe and refractory cases of ILD.

Rituximab has been shown to improve PFT parameters (DLCO and FVC) in patients with refractory myositisrelated ILD (especially ASSD). This association is limited to a few case reports/series ${ }^{51-53}$.

\section{MDA-5 Antibody Seropositivity}

As previously mentioned, MDA-5 Antibody seropositivity portends a rapidly progressive ILD refractory to Cyc, GC and calcineurin inhibitors. Rituximab and Janus
Kinase (JAK) inhibitor tofacitinib have been shown to improve ILD in these patients ${ }^{54-56}$. In an open-label study, tofacitinib usage demonstrated $100 \%$ survival at 6-month interval compared to other immunosuppressants (GC, Cyc, MMF, AZA) in this patient subset ${ }^{57}$. It was also linked with improvement in DLCO and HRCT findings. Similarly, plasma exchange and IVIG administration has shown improvement in this subset of patient population ${ }^{58,59}$, but larger trials are required to better evaluate efficacy of these options.

\section{Rheumatoid Arthritis}

Rheumatoid arthritis has many extra-articular manifestations and lungs are commonly affected; with ILD being the most common form of pulmonary disease $\mathrm{e}^{60,61}$. Rheumatoid arthritis related Interstitial lung disease (RAILD) is a heterogeneous group of different subtypes of ILDs with differing clinical approaches and treatment options. UIP and NSIP are often the most implicated histopathologic and radiographic pattern in RA-ILD ${ }^{62,63}$.

\section{Approach to Therapy}

RA-ILD's treatment modalities continue to be researched on and no optimum therapy guideline is available due to a severe lack of RCTs in this subset of patients. The decision to start immunosuppressive therapy is undertaken after assessment of prognosis, adverse effect of treatment and rate of progression of disease. A pragmatic approach usually involves characterizing disease behavior into categories such as self-limited, reversible, progressive, or irreversible based on serial measurement in predictors of survivals such as DLCO and HRCT picture ${ }^{61,64,65}$.

\section{Glucocorticoid (GC)}

GCs are commonly utilized for initial RA-ILD therapy. Certain features pertain a more favorable response to GCs including younger age, non-UIP histopathologic disease and worsening of HRCT and/or pulmonary function.

If patients fail to show improvement in lung function on GC therapy, an approach involving other immunosuppressants such as MMF, CYC and AZA can be considered after excluding drug toxicity and infection as cause of failure to respond ${ }^{66-68}$. These agents can also be utilized as GC-sparing regimen after initial therapy with GC or in conjugation with them as well in order to minimize adverse effects related with GC.

\section{Methotrexate (MTX) Therapy}

MTX has historically been linked with worsening of pulmonary fibrosis ${ }^{69}$, but newer data suggests that MTX may in fact delay the onset of RA-ILD ${ }^{70}$. Thus, clinically MTX therapy is used if indicated for underlying joint disease.

\section{Progressive Fibrosing ILD}

Patients who have $>10 \%$ lung involvement in fibrosis 
or have feature of extensive reticulation with traction bronchiectasis are defined as to having progressive fibrosing ILD. This form of ILD is a broader term which can be seen with other CTD-ILDs as well and is characterized by progressive decline in lung function (decline in FVC/ DLCO $>10 \%$ ) despite appropriate therapy. Nintedanib has been studied in RA-ILD patients with progressive fibrosis and shows improvement and stability in lung function ${ }^{71}$. However, lack of individualized trials on just RA-ILD patients necessitate continuing research.

Pirfenidone is currently being studied for RA-ILD management in a phase 2 clinical trial ${ }^{72}$. These results and studies on other newer options will direct the course of future treatment options.

\section{Newer Options}

Abatacept, Rituximab and Tocilizumab have all shown modest control of RA-ILD in retrospective studies ${ }^{73-76}$, however there is a need for larger clinical trials to formally assess the efficacy of these treatment options.

\section{Sjogren's Syndrome (SS)}

Sjogren's Syndrome involves lymphocytic infiltration of salivary and lacrimal glands resulting in their destruction. SS related ILD is often the most common manifestation of associated lung disease with NSIP being the most found pattern. UIP is occasionally observed and even though LIP is rarely observed, it is classically associated with $\mathrm{SS}^{77}$.

\section{Approach to Therapy}

Asymptomatic patients can be monitored over a course of months - years with serial PFTs and HRCT to document any worsening in clinical picture as primary SS-ILD often progresses more slowly. For symptomatic patients with worsening symptoms and/or deteriorating HRCT or PFT parameters treatment is usually indicated ${ }^{78}$. Though it needs to be noted that treatment options are usually empiric and there are no formal guidelines available specific for SS related ILD treatment as data is limited to a few case reports/series.

\section{Initial Agent}

GCs are frequently used as initial therapy, response to GCs and GC-sparing agents is usually good with CYC reserved for more severe cases. MMF or AZA can be used to minimize steroid-related side effects and to improve response to $\mathrm{GC}^{67,79,80}$. Patients with radiologic/ histopathologic evidence of NSIP, LIP or OP seem to show a better response to immunosuppressive treatment than those who show evidence of a UIP pattern.

\section{Refractory Disease}

Few case reports and case series have shown improvement and stability in lung function with use of Rituximab ${ }^{81-85}$, thus this option can be utilized for SS-ILD patients refractory to GC and conventional immunosuppressant therapy, though it should be noted that larger trials are still required.

Tocilizumab usage improved SS-ILD in one case of steroid resistant $\mathrm{OP}^{86}$.

\section{Systemic Lupus Erythematosus (SLE)}

SLE is an autoimmune disorder characterized by autoantibodies against nuclear antigens and immune complex deposition in various organs. NSIP is the most common ILD pattern found in SLE patients with OP and LIP pattern being occasionally described ${ }^{87}$. It should be noted that incidence of ILD in SLE is overall relatively low.

Therapeutic options for SLE-ILD are mostly dependent on anecdotes as no clinical trial has been done specifically for this subset. Glucocorticoids are usually the initial agents used ${ }^{88}$, with MMF and AZA acting as steroid sparing agents $^{89}$. CYC and Rituximab may be used for cases resistant to conventional treatment options but evidence is limited due to lack of controlled trials ${ }^{90}$.

\section{Mixed Connective Tissue Disease (MCTD)}

MCTD is defined as an overlapping condition with clinical features of SSc, SLE, RA, and IIM.

Lung involvement in MCTD is usually like SSc related ILD. There have been no controlled studies assessing treatment options in specifically patient with MCTD.

Similar to treatment of ILD related to CTDs, immunosuppressive therapy can be utilized with combination of GCs with AZA and MMF as steroid sparing options ${ }^{91-93}$, with CYC reserved for refractory cases.

\section{Conclusion}

ILD is the most serious manifestation of lung disease associated with CTDs and is often the cause of death in these patients. It imposes a significant clinical challenge to rheumatologists and pulmonologists due to many factors. Some of these factors include lack of large clinical trials demonstrating efficacy of treatment options, unpredictable nature of the disease, lack of clear underlying histopathologic diagnosis and grim prognosis of overall condition.

Therapeutic approach for treatment of CTD-ILD is obtained from mostly retrospective or inconsistent data, apart from approach for SSc related ILD. Therefore, a lot of times clinicians paint every CTD-ILD with the same brush and use similar treatment options. This undermines the diversity in types, pathogenesis, and therapy response among different types of ILD.

Clinically, treatment approach is based on initial 
immunosuppression with glucocorticoids and immunosuppressive regimens, mainly CYC, AZA and MMF, forming the steroid sparing group. Even though strong evidence is still not substantiated, Rituximab is considered a strong contender for both initial therapy as well as for refractory disease ${ }^{36}$.

More recent clinical trials have introduced an array of newer options for CTD-ILD treatment including Nintedanib and Tocilizumab amongst others ${ }^{94,95}$. Having said that, larger controlled trials are still required to formulate treatment guidelines for CTD-ILDs.

\section{References}

1. Takizawa H, Suzuki N, Yanagawa T, et al. Importance of interstitial lung disease in collagen vascular disease: analysis of outcome. Nihon Kyobu Shikkan Gakkai Zasshi. 1996; 34(11): 1177-81.

2. Fischer $A, d u$ Bois R. Interstitial lung disease in connective tissue disorders. Lancet. 2012; 380(9842): 689-98.

3. Cappelli S, Bellando Randone S, Camiciottoli G, et al. Interstitial lung disease in systemic sclerosis: where do we stand? Eur Respir Rev. 2015; 24(137): 411-9.

4. Douglas WW, Tazelaar HD, Hartman TE, et al. Polymyositisdermatomyositis-associated interstitial lung disease. Am J Respir Crit Care Med. 2001; 164(7): 1182-5.

5. Poletti V, Chilosi M, Olivieri D. Diagnostic invasive procedures in diffuse infiltrative lung diseases. Respiration. 2004; 71(2): 107-19.

6. Halkos ME, Gal AA, Kerendi F, et al. Role of thoracic surgeons in the diagnosis of idiopathic interstitial lung disease. Ann Thorac Surg 2005; 79(6): 2172-9.

7. American Thoracic Society/European Respiratory Society International Multidisciplinary Consensus Classification of the Idiopathic Interstitial Pneumonias. This joint statement of the American Thoracic Society (ATS), and the European Respiratory Society (ERS) was adopted by the ATS board of directors, June 2001 and by the ERS Executive Committee, June 2001. Am J Respir Crit Care Med. 2002; 165(2): 277-304.

8. Nicholson AG. Classification of idiopathic interstitial pneumonias: making sense of the alphabet soup. Histopathology. 2002; 41(5): 38191.

9. Mathai SC, Danoff SK. Management of interstitial lung disease associated with connective tissue disease. Bmj. 2016; 352: h6819.

10. Vij R, Strek ME. Diagnosis and treatment of connective tissue diseaseassociated interstitial lung disease. Chest. 2013; 143(3): 814-24.

11. Suliman YA, Dobrota R, Huscher D, et al. Brief Report: Pulmonary Function Tests: High Rate of False-Negative Results in the Early Detection and Screening of Scleroderma-Related Interstitial Lung Disease. Arthritis Rheumatol. 2015; 67(12): 3256-61.

12. Fischer A, Swigris JJ, Groshong SD, et al. Clinically significant interstitial lung disease in limited scleroderma: histopathology, clinical features, and survival. Chest. 2008; 134(3): 601-5.

13. Steen VD, Medsger TA, Jr. Severe organ involvement in systemic sclerosis with diffuse scleroderma. Arthritis Rheum. 2000; 43(11): 2437-44.

14. Bouros D, Wells AU, Nicholson AG, et al. Histopathologic subsets of fibrosing alveolitis in patients with systemic sclerosis and their relationship to outcome. Am J Respir Crit Care Med. 2002; 165(12): 1581-6.

15. Moore OA, Goh N, Corte T, et al. Extent of disease on high-resolution computed tomography lung is a predictor of decline and mortality in systemic sclerosis-related interstitial lung disease. Rheumatology (Oxford). 2013; 52(1): 155-60.

16. Goh NS, Desai SR, Veeraraghavan S, et al. Interstitial lung disease in systemic sclerosis: a simple staging system. Am J Respir Crit Care Med. 2008; 177(11): 1248-54

17. Morisset J, Vittinghoff E, Elicker BM, et al. Mortality Risk Prediction in Scleroderma-Related Interstitial Lung Disease: The SADL Model. Chest. 2017; 152(5): 999-1007.

18. Simeón-Aznar CP, Fonollosa-Plá V, Tolosa-Vilella C, et al. Effect of mycophenolate sodium in scleroderma-related interstitial lung disease. Clin Rheumatol. 2011; 30(11): 1393-8.

19. Volkmann ER, Tashkin DP, Li N, et al. Mycophenolate Mofetil Versus Placebo for Systemic Sclerosis-Related Interstitial Lung Disease: An Analysis of Scleroderma Lung Studies I and II. Arthritis Rheumatol. 2017; 69(7): 1451-60.

20. Tashkin DP, Roth MD, Clements PJ, et al. Mycophenolate mofetil versus oral cyclophosphamide in scleroderma-related interstitial lung disease (SLS II): a randomised controlled, double-blind, parallel group trial. Lancet Respir Med. 2016; 4(9): 708-19.

21. Goldin J, Elashoff R, Kim HJ, et al. Treatment of sclerodermainterstitial lung disease with cyclophosphamide is associated with less progressive fibrosis on serial thoracic high-resolution CT scan than placebo: findings from the scleroderma lung study. Chest. 2009; 136(5): 1333-40.

22. Kowal-Bielecka O, Fransen J, Avouac J, et al. Update of EULAR recommendations for the treatment of systemic sclerosis. Ann Rheum Dis. 2017; 76(8): 1327-39.

23. Hoyles RK, Ellis RW, Wellsbury J, et al. A multicenter, prospective, randomized, double-blind, placebo-controlled trial of corticosteroids and intravenous cyclophosphamide followed by oral azathioprine for the treatment of pulmonary fibrosis in scleroderma. Arthritis Rheum. 2006; 54(12): 3962-70.

24. Steen VD, Medsger TA, Jr. Case-control study of corticosteroids and other drugs that either precipitate or protect from the development of scleroderma renal crisis. Arthritis Rheum. 1998; 41(9): 1613-9.

25. Bérezné A, Ranque B, Valeyre D, et al. Therapeutic strategy combining intravenous cyclophosphamide followed by oral azathioprine to treat worsening interstitial lung disease associated with systemic sclerosis: a retrospective multicenter open-label study. J Rheumatol. 2008; 35(6): 1064-72.

26. Oldham JM, Lee C, Valenzi E, et al. Azathioprine response in patients with fibrotic connective tissue disease-associated interstitial lung disease. Respir Med. 2016; 121: 117-22.

27. De Lauretis A, Sestini P, Pantelidis P, et al. Serum interleukin 6 is predictive of early functional decline and mortality in interstitial lung disease associated with systemic sclerosis. J Rheumatol. 2013; 40(4): $435-46$.

28. Roofeh D, Lescoat A, Khanna D. Treatment for systemic sclerosisassociated interstitial lung disease. Curr Opin Rheumatol. 2021; 33(3): 240-8.

29. Khanna D, Lin CJF, Furst DE, et al. Tocilizumab in systemic sclerosis: a randomised, double-blind, placebo-controlled, phase 3 trial. Lancet Respir Med. 2020; 8(10): 963-74.

30. Khanna D, Denton CP, Lin CJF, et al. Safety and efficacy of subcutaneous tocilizumab in systemic sclerosis: results from the open-label period of a phase II randomised controlled trial (faSScinate). Ann Rheum Dis. 2018; 77(2): 212-20.

31. Richeldi L, du Bois RM, Raghu G, et al. Efficacy and safety of nintedanib in idiopathic pulmonary fibrosis. N Engl J Med. 2014; 370(22): 207182. 
32. Distler O, Highland KB, Gahlemann M, et al. Nintedanib for Systemic Sclerosis-Associated Interstitial Lung Disease. N Engl J Med. 2019; 380(26): 2518-28.

33. Acharya N, Sharma SK, Mishra D, et al. Efficacy and safety of pirfenidone in systemic sclerosis-related interstitial lung disease-a randomised controlled trial. Rheumatol Int. 2020; 40(5): 703-10.

34. Miura Y, Saito T, Fujita $\mathrm{K}$, et al. Clinical experience with pirfenidone in five patients with scleroderma-related interstitial lung disease. Sarcoidosis Vasc Diffuse Lung Dis. 2014; 31(3): 235-8.

35. Hoffmann-Vold AM, Maher TM, Philpot EE, et al. Assessment of recent evidence for the management of patients with systemic sclerosisassociated interstitial lung disease: a systematic review. ERJ Open Res. 2021.

36. Goswami RP, Ray A, Chatterjee M, et al. Rituximab in the treatment of systemic sclerosis-related interstitial lung disease: a systematic review and meta-analysis. Rheumatology (Oxford). 2021; 60(2): 557 67.

37. Rossi D, Sciascia S, Cecchi I, et al. A 3-Year Observational Study of Patients with Progressive Systemic Sclerosis Treated with an Intensified B Lymphocyte Depletion Protocol: Clinical and Immunological Response. J Clin Med. 2021.

38. Vojinovic T, Cavazzana I, Ceruti P, et al. Predictive Features and Clinical Presentation of Interstitial Lung Disease in Inflammatory Myositis. Clin Rev Allergy Immunol. 2021; 60(1): 87-94.

39. Gutsche M, Rosen GD, Swigris JJ. Connective Tissue Disease-associated Interstitial Lung Disease: A review. Curr Respir Care Rep. 2012; 1 224-32.

40. Labrador-Horrillo M, Martinez MA, Selva-O'Callaghan A, et al. AntiMDA5 antibodies in a large Mediterranean population of adults with dermatomyositis. J Immunol Res. 2014; 2014: 290797.

41. Sato S, Hoshino K, Satoh T, et al. RNA helicase encoded by melanoma differentiation-associated gene 5 is a major autoantigen in patients with clinically amyopathic dermatomyositis: Association with rapidly progressive interstitial lung disease. Arthritis Rheum. 2009; 60(7): 2193-200.

42. Yoshifuji H, Fujii T, Kobayashi S, et al. Anti-aminoacyl-tRNA synthetase antibodies in clinical course prediction of interstitial lung disease complicated with idiopathic inflammatory myopathies. Autoimmunity. 2006; 39(3): 233-41.

43. Fujisawa T, Suda T, Nakamura Y, et al. Differences in clinical features and prognosis of interstitial lung diseases between polymyositis and dermatomyositis. J Rheumatol. 2005; 32(1): 58-64.

44. Bonnefoy O, Ferretti G, Calaque 0, et al. Serial chest CT findings in interstitial lung disease associated with polymyositisdermatomyositis. Eur J Radiol. 2004; 49(3): 235-44.

45. Moghadam-Kia S, Oddis CV, Sato S, et al. Antimelanoma Differentiationassociated Gene 5 Antibody: Expanding the Clinical Spectrum in North American Patients with Dermatomyositis. J Rheumatol. 2017; 44(3): 319-25.

46. Huapaya JA, Silhan L, Pinal-Fernandez I, et al. Long-Term Treatment With Azathioprine and Mycophenolate Mofetil for Myositis-Related Interstitial Lung Disease. Chest. 2019; 156(5): 896-906.

47. Mira-Avendano IC, Parambil JG, Yadav R, et al. A retrospective review of clinical features and treatment outcomes in steroid-resistant interstitial lung disease from polymyositis/dermatomyositis. Respir Med. 2013; 107(6): 890-6.

48. Sharma N, Putman MS, Vij R, et al. Myositis-associated Interstitial Lung Disease: Predictors of Failure of Conventional Treatment and Response to Tacrolimus in a US Cohort. J Rheumatol. 2017; 44(11): 1612-8.
49. Kurita T, Yasuda S, Oba K, et al. The efficacy of tacrolimus in patients with interstitial lung diseases complicated with polymyositis or dermatomyositis. Rheumatology (Oxford). 2015; 54(1): 39-44.

50. Connors GR, Christopher-Stine L, Oddis CV, et al. Interstitial lung disease associated with the idiopathic inflammatory myopathies: what progress has been made in the past 35 years? Chest. 2010; 138(6): 1464-74

51. Marie I, Dominique S, Janvresse A, et al. Rituximab therapy for refractory interstitial lung disease related to antisynthetase syndrome. Respiratory medicine. 2012; 106(4): 581-7.

52. Dasa 0, Ruzieh M, Oraibi O. Successful Treatment of Life-Threatening Interstitial Lung Disease Secondary to Antisynthetase Syndrome Using Rituximab: A Case Report and Review of the Literature. Am J Ther. 2016; 23(2): e639-45.

53. Keir GJ, Maher TM, Ming D, et al. Rituximab in severe, treatmentrefractory interstitial lung disease. Respirology. 2014; 19(3): 353-9.

54. So H, Wong VTL, Lao VWN, et al. Rituximab for refractory rapidly progressive interstitial lung disease related to anti-MDA5 antibody-positive amyopathic dermatomyositis. Clin Rheumatol. 2018; 37(7): 1983-9.

55. Kurasawa K, Arai S, Namiki Y, et al. Tofacitinib for refractory interstitial lung diseases in anti-melanoma differentiation-associated 5 gene antibody-positive dermatomyositis. Rheumatology (Oxford). 2018; 57(12): 2114-9.

56. Hornig J, Weinhage T, Schmidt LH, et al. Response of dermatomyositis with lung involvement to Janus kinase inhibitor treatment. Z Rheumatol. 2018; 77(10): 952-7.

57. Chen Z, Wang X, Ye S. Tofacitinib in Amyopathic DermatomyositisAssociated Interstitial Lung Disease. The New England journal of medicine. 2019; 381(3): 291-3.

58. Endo Y, Koga T, Suzuki T, et al. Successful treatment of plasma exchange for rapidly progressive interstitial lung disease with antiMDA5 antibody-positive dermatomyositis: A case report. Medicine (Baltimore). 2018; 97(15): e0436.

59. Suzuki Y, Hayakawa H, Miwa S, et al. Intravenous immunoglobulin therapy for refractory interstitial lung disease associated with polymyositis/dermatomyositis. Lung. 2009; 187(3): 201-6.

60. Tanoue LT. Pulmonary manifestations of rheumatoid arthritis. Clin Chest Med. 1998; 19(4): 667-85, viii.

61. Lake F, Proudman S. Rheumatoid arthritis and lung disease: from mechanisms to a practical approach. Semin Respir Crit Care Med. 2014; 35(2): 222-38.

62. Kelly CA, Saravanan V, Nisar M, et al. Rheumatoid arthritis-related interstitial lung disease: associations, prognostic factors and physiological and radiological characteristics--a large multicentre UK study. Rheumatology (Oxford). 2014; 53(9): 1676-82.

63. Lee HK, Kim DS, Yoo B, et al. Histopathologic pattern and clinical features of rheumatoid arthritis-associated interstitial lung disease. Chest. 2005; 127(6): 2019-27.

64. Yunt ZX, Chung JH, Hobbs S, et al. High resolution computed tomography pattern of usual interstitial pneumonia in rheumatoid arthritis-associated interstitial lung disease: Relationship to survival. Respir Med. 2017; 126: 100-4.

65. Cottin V. Pragmatic prognostic approach of rheumatoid arthritisassociated interstitial lung disease. Eur Respir J. 2010; 35(6): 1206-8.

66. Saketkoo LA, Espinoza LR. Rheumatoid arthritis interstitial lung disease: mycophenolate mofetil as an antifibrotic and disease-modifying antirheumatic drug. Arch Intern Med. 2008; 168(15): 1718-9.

67. Fischer A, Brown KK, Du Bois RM, et al. Mycophenolate mofetil improves lung function in connective tissue disease-associated interstitial lung disease. J Rheumatol. 2013; 40(5): 640-6. 
68. Schupp JC, Köhler T, Müller-Quernheim J. Usefulness of Cyclophosphamide Pulse Therapy in Interstitial Lung Diseases. Respiration. 2016; 91(4): 296-301.

69. Quah E, Amoasii C, Mudawi T, et al. Systematic literature review investigating whether methotrexate causes chronic pulmonary fibrosis. Future Healthc J. 2019; 6(Suppl 2): 4.

70. Kiely P, Busby AD, Nikiphorou E, et al. Is incident rheumatoid arthritis interstitial lung disease associated with methotrexate treatment? Results from a multivariate analysis in the ERAS and ERAN inception cohorts. BMJ Open. 2019; 9(5): e028466.

71. Flaherty KR, Wells AU, Cottin V, et al. Nintedanib in Progressive Fibrosing Interstitial Lung Diseases. The New England journal of medicine. 2019; 381(18): 1718-27.

72. Solomon JJ, Danoff SK, Goldberg HJ, et al. The Design and Rationale of the Trail1 Trial: A Randomized Double-Blind Phase 2 Clinical Trial of Pirfenidone in Rheumatoid Arthritis-Associated Interstitial Lung Disease. Adv Ther. 2019; 36(11): 3279-87.

73. Fui A, Bergantini L, Selvi E, et al. Rituximab therapy in interstitial lung disease associated with rheumatoid arthritis. Intern Med J. 2020; 50(3): 330-6.

74. Kurata I, Tsuboi H, Terasaki M, et al. Effect of Biological Diseasemodifying Anti-rheumatic Drugs on Airway and Interstitial Lung Disease in Patients with Rheumatoid Arthritis. Intern Med. 2019; 58(12): 1703-12.

75. Manfredi A, Cassone G, Furini F, et al. Tocilizumab therapy in rheumatoid arthritis with interstitial lung disease: a multicentre retrospective study. Intern Med J. 2020; 50(9): 1085-90.

76. Fernández-Díaz C, Castañeda S, Melero-González RB, et al. Abatacept in interstitial lung disease associated with rheumatoid arthritis: national multicenter study of 263 patients. Rheumatology (Oxford). 2020; 59(12): 3906-16.

77. Roca F, Dominique S, Schmidt J, et al. Interstitial lung disease in primary Sjögren's syndrome. Autoimmun Rev. 2017; 16(1): 48-54.

78. Robles-Perez A, Molina-Molina M. Treatment Considerations of Lung Involvement in Rheumatologic Disease. Respiration. 2015; 90(4): 265-74.

79. Parambil JG, Myers JL, Lindell RM, et al. Interstitial lung disease in primary Sjögren syndrome. Chest. 2006; 130(5): 1489-95.

80. Deheinzelin D, Capelozzi VL, Kairalla RA, et al. Interstitial lung disease in primary Sjögren's syndrome. Clinical-pathological evaluation and response to treatment. American journal of respiratory and critical care medicine. 1996; 154(3 Pt 1): 794-9.

81. Saraux A, Pers JO, Devauchelle-Pensec V. Treatment of primary Sjögren syndrome. Nat Rev Rheumatol. 2016; 12(8): 456-71.
82. Both T, Dalm VA, van Hagen PM, et al. Reviewing primary Sjögren's syndrome: beyond the dryness - From pathophysiology to diagnosis and treatment. Int J Med Sci. 2017; 14(3): 191-200.

83. Swartz MA, Vivino FB. Dramatic reversal of lymphocytic interstitial pneumonitis in Sjögren's syndrome with rituximab. J Clin Rheumatol. 2011; 17(8): 454.

84. Gottenberg JE, Cinquetti G, Larroche C, et al. Efficacy of rituximab in systemic manifestations of primary Sjogren's syndrome: results in 78 patients of the AutoImmune and Rituximab registry. Ann Rheum Dis. 2013; 72(6): 1026-31.

85. Bellan M, Patrucco F, Barone-Adesi F, et al. Targeting CD20 in the treatment of interstitial lung diseases related to connective tissue diseases: A systematic review. Autoimmun Rev. 2020; 19(2): 102453.

86. Justet $\mathrm{A}$, Ottaviani $\mathrm{S}$, Dieude $\mathrm{P}$, et al. Tocilizumab for refractory organising pneumonia associated with Sjogren's disease. BMJ case reports. 2015.

87. Wallace B, Vummidi D, Khanna D. Management of connective tissue diseases associated interstitial lung disease: a review of the published literature. Curr Opin Rheumatol. 2016; 28(3): 236-45.

88. Weinrib L, Sharma OP, Quismorio FP, Jr. A long-term study of interstitial lung disease in systemic lupus erythematosus. Semin Arthritis Rheum. 1990; 20(1): 48-56.

89. Nawata M, Nagayasu A, Fujita Y, et al. Severe pulmonary arterial hypertension and interstitial pneumonia related to systemic lupus erythematosus successfully treated with mycophenolate mofetil: A novel case report. Lupus. 2020; 29(14): 1955-60.

90. Lim SW, Gillis D, Smith W, et al. Rituximab use in systemic lupus erythematosus pneumonitis and a review of current reports. Intern Med J. 2006; 36(4): 260-2.

91. Reiseter S, Gunnarsson R, Corander J, et al. Disease evolution in mixed connective tissue disease: results from a long-term nationwide prospective cohort study. Arthritis Res Ther. 2017; 19(1): 284

92. Graney BA, Fischer A. Advocating for early interstitial lung disease detection in mixed connective tissue disease. Rheumatology (Oxford). 2018; 57(2): 204-5.

93. Koo SM, Uh ST. Treatment of connective tissue disease-associated interstitial lung disease: the pulmonologist's point of view. Korean J Intern Med. 2017; 32(4): 600-10.

94. Wells AU, Flaherty KR, Brown KK, et al. Nintedanib in patients with progressive fibrosing interstitial lung diseases-subgroup analyses by interstitial lung disease diagnosis in the INBUILD trial: a randomised, double-blind, placebo-controlled, parallel-group trial. Lancet Respir Med. 2020; 8(5): 453-60.

95. Roofeh D, Lin CJF, Goldin J, et al. Tocilizumab Prevents Progression of Early Systemic Sclerosis-Associated Interstitial Lung Disease. Arthritis Rheumatol. 2021; 73(7): 1301-10. 